\title{
Abnormal nitrogen metabolism in uremia
}

\author{
William. E. Mitch, Y. Hara* \\ Renal Division, Emory University School of Medicine, Atlanta, Georgia, Tokyo Women's Medical College, Tokyo*
}

Abnormal nitrogen and, by implication, protein metabolism is a common feature associated with loss of renal function and many investigators attribute the morbidity associated with uremia to loss of protein stores. For example, Kopple has noted that between 1976-1984, every publication concerning the nutritional status of patients entering chronic dialysis suggests loss of lean body mass ${ }^{1)}$. Moreover, in patients with acute renal failure (ARF), negative nitrogen balance, accelerated accumulation of nitrogenous waste products and abnormal anthropometry indicate impaired protein conservation ${ }^{2}$. Despite the clinical importance of protein wasting in uremia, it is not known why it occurs, what factor(s) in uremia initiate changes in protein metabolism or how metabolic changes are mediated. Clearly, it would be advantageous to identify the mechanisms causing abnormal protein metabolism because of its effects on morbidity and mortality in patients with uremia $^{1 \sim 3)}$.

Factors causing loss of protein mass in uremic patients could include an inadequate diet, activation of protein catabolism and/or suppression of protein synthesis. Ensuring that nutrient requirements are met has become even more critical because many patients with chronic renal failure (CRF) are prescribed a low protein diet in an attempt to slow the loss of renal insufficiency ${ }^{4}$. Since the daily turnover of body protein is quite large, even small changes in protein synthesis or degradation will lead to loss of lean body mass over extended periods. Regarding overall protein metabolism, it has been estimated that a normal $70 \mathrm{~kg}$ adult synthesizes and degrades about $320 \mathrm{~g}$ protein/day. Consequently, even a small percentage decrease in protein synthesis and/or increase in degradation lasting for several months would lead to a severe loss of lean body mass ${ }^{5}$.

\section{CATABOLIC FACTORS IN UREMIA}

Insulin resistance

Certain hormonal abnormalities associated with uremia, such as insulin resistance ${ }^{6,7)}$, could change the control of protein synthesis and/or degradation and cause loss of lean body mass. If the ability of insulin to stimulate amino acid uptake by muscle were impaired, intracellular pools of amino acids might become depleted or an abnormal distribution of amino acids might occur, impairing protein synthesis. Maroni et al. examined the impact of uremia on amino acid uptake by measuring the function of the three major transporters of neutral amino acids in muscle, Systems A, ASC and $L^{8,9}$. Methylaminoisobutyric acid, a probe transported specifically by System A, was used to show that transport by System A in muscle is impaired by ARF in the absence of insulin ${ }^{8}$. The response to pharmacologic concentrations of insulin also was blunted in muscle of ARF rats. On the other hand, insulin sensitivity and stimulation of System A transport by physiologic concentrations of insulin was preserved. It also was shown that uptake through the other major, neutral amino acid transporters in muscle, Systems ASC and L was unimpaired by $\mathrm{ARF}^{9)}$. It was concluded that impaired insulin-stimulated amino acid uptake does not have a major role in causing abnormal muscle protein turnover.

Insulin-stimulated protein synthesis in muscle is subnormal in both experimental acute and chronic uremia $^{10,11)}$. Unfortunately, the mechanism for this defect is not understood. There seems to be no abnormality of insulin binding in uremia, so that it has been postulated that a post-receptor defect must be present ${ }^{6}$. The dose-response relationships between insulin concentration and different parameters of muscle glucose metabolism including glucose uptake, glycogen synthesis and glucose oxidation were found 
to be consistent with a post-receptor defect. May et al. reported that the insulin concentration causing halfmaximal stimulation of each aspect of glucose metabolism was not different in muscles of control and ARF rats ${ }^{7)}$ indicating that insulin-binding characteristics must be normal ${ }^{12}$. On the other hand, the doseresponse curves also indicated that the maximal rate of each metabolic function was lower in muscles of ARF rats. Moreover, the ability of insulin to activate the cytosolic enzyme, glycogen synthase, in muscle of ARF rats was clearly subnormal. Both of these findings are consistent with the presence of a post-receptor defect in the action of insulin and by analogy, it seems likely that the subnormal protein synthesis in response to insulin in the same model is most likely due to a post-receptor defect ${ }^{10)}$. It should be emphasized that in both ARF and CRF, the degree of abnormal muscle protein synthesis was small compared to the increase in protein degradation ${ }^{10,11}$.

Defective energy metabolism

A potential mechanism linking defective insulin action to increased protein breakdown might be abnormal energy metabolism. When the proportion of insulin-mediated glucose uptake shunted into glycolysis or lactate release in muscle was compared to the rate of protein degradation in the same muscle, a positive correlation was uncovered ${ }^{7,10)}$. These results suggest that in uremia, the increase in the ratio of glycolysis to glucose uptake leads to stimulation of protein breakdown. A similar positive correlation was found in investigations of muscles of fed or starved normal rats, or rats responding to the catabolic stress of thermal injury ${ }^{13,14)}$. To study this crrelation further, we used two methods of changing glucose metabolism to determine if the relationship would be uncoupled. In the first study, muscles from normal rats were incubated with vanadate because we had found that vanadate exerted insulin-like changes in glucose metabolism including a stimulation of glucose uptake and glycolysis ${ }^{15}$. Although glucose metabolism was altered, vanadate did not change the proportion of glucose uptake that was shunted into glycolysis and we predicted that proteolysis would not be stimulated $^{13)}$. In fact, neither protein synthesis nor protein degradation were changed by incubating muscles with vanadate ${ }^{15)}$. In a second study, Clark et al. incubated muscles with dichloroacetate to stimulate pyruvate dehydrogenase and alter lactate production. Dichloroacetate did change the proportion of muscle glucose uptake shunted into glycolysis (or lactate production) but unexpectedly, protein degradation was unaffected ${ }^{16}$. Results obtained with dichloroacetate must mean that a change in glycolysis does not directly stimulate proteolysis; it remains possible, however, that the close correlation between increased muscle glycolysis and protein breakdown is caused by activation of parallel pathways stimulated by insulin binding to its receptor.

Other evidence for a link between abnormal energy metabolism and protein turnover in uremia was reported by $\mathrm{Li}$ and Wassner ${ }^{17}$. Using the isolated perfused hindquarter, they found that diminished body fat stores in CRF were closely correlated with increased net protein degradation in muscle. Others have shown that in rats with $\mathrm{CRF}$, glucose metabolism by adipocytes is impaired because of a factor present in serum ${ }^{18}$. Whether diminished fat stores in CRF are linked to excessive utilization of lipids to provide the energy necessary for protein breakdown in muscle is unknown.

An alternative explanation for increased protein catabolism in uremia would be that a common abnormality (e.g. a circulating factor or a biochemical defect) in muscle, adipocytes, and possibly other tissues leads to accelerated breakdown of protein, fat, glucose, etc. For example, McCaleb et al. reported that insulin resistance could be induced in normal adipocytes by incubating them with serum obtained from dialysis patients ${ }^{18}$ and Druml et al. recently found that amino acid transport in adipocytes of CRF rats is subnormal and is closely correlated with defects in cation transport ${ }^{19}$. Defective erythrocyte cation transport was noted by Welt and associates in 1964 in studies of dialysis patients ${ }^{20)}$. The demonstration by Druml et al. that abnormal cation transport is present in adipocytes and skeletal muscle and is closely linked to impaired sodium-dependent amino acid transport suggests that changes in intracellular ions might play an important role in the control of cellular metabolism in uremia. Since abnormal glucose, lipid and protein metabolism are interrelated, a defect in the control of intracellular ions could contribute to 
loss of protein stores.

Recently, a mechanism for abnormal cellular ion transport in uremia has been proposed. Kelly and associates found that the plasma of normal humans contains substances that exhibit digitalis-like activity ; these substances inhibit NaK-ATPase activity in vitro, displace ouabain from NaK-ATPase, and impair normal cation flux in erythrocytes ${ }^{21}$. Subsequently, Kelly et al. showed that unsaturated fatty acids and lysophospholipids in plasma could account for all of these properties ${ }^{22)}$ and that the same factors are present at somewhat higher concentrations in the plasma of uremic patients ${ }^{23}$. Indirect evidence suggested that the inhibitory effect of plasma lipids does not involve direct binding to the ouabain-binding site of NaK-ATPase, but instead could be caused by a change in membrane composition and function leading to secondary inhibition of enzyme activity. If this were true, changes in membrane function might provide a link between defective cation transport and abnormal amino acid transport in adipocytes from CRF rats $^{19)}$. Moreover, changes in membrane function also might affect other transporters and increase intracellular hydrogen ion content.

Metabolic acidosis

A major cause of excessive muscle proteolysis in rats with experimental uremia is metabolic acidosis. When rats with normal renal function were made acidotic by feeding ammonium chloride or hydrochloric acid, both glucocorticoid production and muscle protein degradation were sharply stimulated ${ }^{24}$. May et al. showed that stimulation of muscle protein breakdown by acidosis required excessive glucocorticoid production ; proteolysis was not increased if rats were first adrenalectomized, and then rendered acidotic, nor was it increased if normal muscles were incubated in acidified media ${ }^{24)}$, The importance of these results to uremia is that rats with $\mathrm{CRF}$ and a milder degree of metabolic acidosis (plasma bicarbonate $<21 \mathrm{mM}$ ) also exhibited increased muscle protein breakdown ${ }^{11}$. In the latter studies, May et al. were able to separate the proteolytic effects of metabolic acidosis from the degree of azotemia, and showed that protein breakdown was not caused by a higher rate of glucocorticoid production unless acidosis was present. Thus, metabolic acidosis, when combined with an increase in glucocorticoid production, stimulates muscle protein breakdown.

Influence of branched-chain amino acids

A potential mechanism causing abnormal muscle protein turnover in uremia could be linked to excessive catabolism of leucine and its ketoacid, alphaketoisocaproate (KIC). In incubated muscles, leucine stimulates protein synthesis while KIC inhibits protein breakdown ; the inhibitory effect of KIC is not shared by valine, isoleucine or their alpha-ketoacids (25) . These findings may have clinical relevance since infusion of KIC into starving obese subjects was found to improve their nitrogen balance ${ }^{26)}$. The improvement in nitrogen balance in these subjects was attributable almost entirely to a decrease in the rate of urea production, suggesting that KIC inhibited net protein degradation, just as in incubated muscle. The relevance of these findings to the increased protein catabolism of uremia is that metabolic acidosis stimulates the activity of branched-chain ketoacid dehydrogenase in muscle and therefore, increases the degradation of KIC and branched-chain amino acids ${ }^{27}$.

The milder metabolic acidosis occurring as a complication of $\mathrm{CRF}$ also increases branched-chain amino acid catabolism. Hara et al. compared pairfed, shamoperated rats with CRF rats that had a mild metabolic acidosis. The CRF rats exhibited accelerated decarboxylation of both valine and leucine in muscle, but when the metabolic acidosis was corrected by feeding bicarbonate, the accelerated amino acid decarboxylation was eliminated, and plasma and muscle levels of leucine rose ${ }^{28)}$. In this same experimental model, protein breakdown was brought to a normal rate $^{11}$.Thus, the metabolic acidosis of uremia will raise the dietary requirement for valine, isoleucine and leucine and may also change the control of protein turnover. The clinical association is that correction of metabolic acidosis in patients with CRF can improve their nitrogen balance ${ }^{29)}$.

In summary, there is evidence that the control of nitrogen metabolism is abnormal in uremia because muscle protein catabolism is accelerated. The catabolic effect of uremia is caused by impaired insulin-stimulated protein synthesis, and more importantly, by increased muscle protein degradation. The latter can be eliminated by correcting the metabolic 
acidosis of uremia. Further work will be necessary to determine if changes in amino acid metabolism caused by the acidosis of uremia accounts for defects in protein metabolism and ultimately, loss of lean body mass.

\section{REFERENCES}

1) Kopple J D : Causes of catabolism and wasting in acute or chronic renal failure, In "Nephrology", ed, Robinson RR, Springer-Verlag, New York, 1984

2) Mitch WE, Wilmore D: Metabolic and nutritional factors in the treatment of acute renal failure, In "Acute Renal Failure", ed BM Brenner, JM Lazarus, Churchill-Livingstone, New York, 1988

3) Mitch W E, Walser M : Nutritional therapy of the uremic patients, In "The Kidney, Edition III", ed B M Brenner, F C Rector, W B Saunders, New York, 1986

4) Mitch $W E$ : Nutritional therapy and the progression of renal insufficiency, In "Nutrition and the Kidney”, ed WE Mitch, S Klahr, Little Brown and Co Boston, 1988

5) Holliday M A : Protein intake, renal function and growth in chronic renal failure, In "The Progressive Nature of Renal Disease", ed Mitch WE Churchill-Livingstone, New York, 1986

6) DeFronzo R A, Alvestrand A, Smith D, Hendler $\mathrm{R}$, Hendler $\mathrm{E}$, Wahren $\mathrm{J}$ : Insulin resistance in uremia. J Clin Invest $67: 563-568,1981$

7) May R C, Clark A S, Goheer A, Mitch W E : Identification of specific defects in insulin-mediated muscle metabolism in acute uremia. Kidney Int $28: 490-497,1985$

8) Maroni B J, Karapanos G, Mitch W E : System A amino acid transport in incubated muscle: Effects of insulin and acute uremia. Am J Physiol 251 : F 74-F 80, 1986

9) Maroni B J, Karapanos G, Mitch W E : System ASC and Naindependent neutral amino acid transport in muscle of uremic rats. Am J Physiol 251 : F 81-F 86, 1986

10) Clark A S, Mitch W E : Muscle protein turnover and glucose uptake in acutely uremic rats: Effects of insulin and the duration of renal insuffi- ciency. J Clin Invest $72: 836-845,1983$

11) May R C, Kelly R A, Mitch W E : Mechanisms for defects in muscle protein metabolism in rats with chronic uremia: The influence of metabolic acidosis. J Clin Invest 79 : 1099-1103, 1987

12) Kahn $C R$ : Insulin resistance, insulin sensitivity and insulin unresponsiveness: A necessary distinction. Metabolism 27 (Suppl 12) : 1893-1902, 1978

13) Mitch W E, Clark A S, May R C : Relationships between protein degradation and glucose metabolism in skeletal muscle, In "Proc Vth International Congress on Proteolysis", ed Khairallah E, Bond J, Bond W S Alan R Liss New York, 1985

14) Clark A S, Kelly R A, Mitch WE : Systemic response to thermal injury in rats: Increased protein degradation and altered glucose utilization in muscle. J Clin Invest $74: 888-897,1984$

15) Clark A S, Fagan J M, Mitch W E : Selectivity of the insulinlike actions of vanadate on glucose and protein metabolism in skeletal muscle. Biochem $\mathrm{J}$ $232: 273-276,1985$

16) Clark A S, Mitch $W$ E, Goodman M N, Fagan J M, Goheer A, Curnow R T : Dichloroacetate augments insulin-stimulated glycogen synthesis and inhibits glycolysis in rat skeletal muscle. J Clin Invest $79: 588-594,1987$

17) Li J B, Wassner S J : Protein synthesis and degradation in skeletal muscle of chronically uremic rats. Kidney Int $29: 1136-1143,1986$

18) McCaleb M L, Mevorach R, Freeman R B, Izzo M $\mathrm{S}$, Lockwood D H : Induction of insulin resistance in normal adipose tissue by uremic human serum. Kidney Int $25: 416-421,1984$

19) Druml W, Kelly R A, May R C, Mitch W E : Abnormal cation transport in uremia: Mechanisms in adipocytes and skeletal muscle. $\mathrm{J}$ Clin Invest $81: 1197-1203,1988$

20) Welt L G, Sacks J R, McManus T J : An ion transport defect in erythrocytes from uremic patients. Trans Assoc Am Physicians 77 : 169181, 1964

21) Kelly R A, O'Hara D S, Mitch W E, Smith T W : Characterization of digitalis-like factors in human plasma: interactions with NaK-ATPase and crossreactivity with cardiac glycoside-spe- 
cific antibodies. J Biol Chem 260:11396-11405, 1985

22) Kelly R A, O'Hara D S, Mitch W E, Smith T W : Identification of digitalis-like factos in human plasma. J Biol Chem 261 : 11704-11711, 1986

23) Kelly R A, O'Hara D S, Mitch W E, Steinman T I, Goldszer R C, Solomon H S, Smith TW: Endogenous digitalis-like factors in hypertension and chronic renal insufficiency. Kidney Int 30 : 723-729, 1986

24) May R C, Kelly R A, Mitch WE: Metabolic acidosis stimulates protein degradation in rat muscle by a glucocorticoid-dependent mechanism. J Clin Invest $77: 614-621,1986$

25) Mitch W E, Clark A S : Specificity of the effect of leucine and its metabolites on protein degradation in skeletal muscle Biochem J 222: 579-586, 1984

26) Mitch W E, Walser M, Sapir D G : Nitrogen spar- ing induced by leucine compared with that induced by its keto analogue, alphaketoisocaproate, in fasting obese man. J Clin Invest $67: 553-562$, 1981

27) May R C, Hara Y, Kelly R A, Block K P, Buse M G, Mitch WE: Branched-chain amino acid metabolism in rat muscle: Abnormal regulation in acidosis. Am J Physiol 252 : E 712-E 718, 1987

28) Hara Y, May RC, Kelly R A, Mitch WE: Acidosis, not azotemia, stimulates branched -chain amino acid catabolism in uremic rats. Kidney Int 32 : 808-814, 1987

29) Papadoyannakis N J, Stefandis C J, McGeown $\mathrm{M}$ : The effect of correction of metabolic acidosis on nitrogen and potassium balance of patients with chronic renal failure. Am J Clin Nutr 40 : 623627, 1984 